J. Lake Sci. (湖泊科学), 2013, 25(5): 765-774

http: //www. jlakes. org. E-mail : jlakes@niglas.ac.cn

(c) 2013 by Journal of Lake Sciences

\title{
气候变暖下太湖极端洪水的归因探讨
}

\author{
于 革, 郭 娅, 廖梦娜 \\ (中国科学院南京地理与湖泊研究所,南京 210008)
}

\begin{abstract}
摘 要: 全球增温引起的降水变化是否引起极端洪水的增加, 发生在不同气候背景的极端洪水事件可提供不同参照系; 而不同驱动因子下气候、水文数值模拟为认识洪水发生和归因提供了有效途径. 本文结合机理数值模拟和随机统计模拟 两种途径, 针对 $1990 \mathrm{~s}$ 和 $1880 \mathrm{~s}$ 的太湖流域特大洪水, 通过 GCM 气候模拟驱动的流域水文模拟和不确定性的阈值模拟, 分析 19 世纪末和 20 世纪末极端洪水的发生强度和频率的变化, 从而论证极端洪水发生的风险系数. 结果表明, 1990s 的 极端洪水流量 $\left(0.1 \%\right.$ 的极端洪水流量 $\left(Q_{0.1 \%}\right)$ 为 $2929 \sim 3601 \mathrm{~m}^{3} / \mathrm{s}, 0.5 \%$ 的极端洪水流量 $\left(Q_{0.5 \%}\right)$ 为 $\left.1842 \sim 1893 \mathrm{~m}^{3} / \mathrm{s}\right)$ 比工业革命前大气温室气体状况下 $\left(Q_{0.1 \%}\right.$ 为 $2069 \sim 3119 \mathrm{~m}^{3} / \mathrm{s}, Q_{0.5 \%}$ 为 $\left.1436 \sim 1561 \mathrm{~m}^{3} / \mathrm{s}\right)$ 显著增大. 与 19 世纪末相比, 由于太湖流域人类活动改变的流域下垫面在 1999 年特大洪水中引起最大增量占 $35 \%$, 本文模拟和分析的 20 世纪末气候 下的洪水最大增量占 $60 \%$.去除人类活动影响的下垫面变化,估计特大洪水风险的最大增量为 $25 \%$,因此认为 20 世纪末 气候变化引起的太湖极端洪水风险在增加;这将为认识与全球增温相关联的洪水灾害预测预警提供科学依据.
\end{abstract}

关键词: 极端洪水;气候效应;不确定性;流域水文模拟;归因;太湖

\section{Probe on attributions of extreme floods responding to the climate changes in Lake Taihu catchment}

\author{
YU Ge, GUO Ya \& LIAO Mengna \\ (Nanjing Institute of Geography and Limnology, Chinese Academy of Sciences, Nanjing 210008, P. R. China)
}

\begin{abstract}
Extreme floods under different climate systems, the pre-industrial time, and the end of the 20th century can provide good comparisons to examine differences among different mechanisms, while numerical models of the climate and hydrology could provide effective ways to simulate processes of the extreme flood changes and analyze the attributions. The paper applied the numerical modeling and statistical analyses to simulate extreme floods of the 1990s and the 1880s in Lake Taihu, driving by different scenarios-climate simulations from four GCMs, and analyzed the flood frequency differences between the end of the 19th and 20th centuries. Results show that extreme flood discharges in $1990 \mathrm{~s}\left(Q_{0.1 \%} 2929-3601 \mathrm{~m}^{3} / \mathrm{s}\right.$ and $\left.Q_{0.5 \%} 1842-1893 \mathrm{~m}^{3} / \mathrm{s}\right)$ have exceed statistically those during pre-industrial time $\left(Q_{0.1 \%} 2069-3119 \mathrm{~m}^{3} / \mathrm{s}\right.$ and $\left.Q_{0.5 \%} 1436-1561 \mathrm{~m}^{3} / \mathrm{s}\right)$. Comparing these floods with those at the end of the 19th century, the highest extreme-flood risks produced by land surface changes due to human activities were $35 \%$ increases, and the total highest risks were $60 \%$ increases in the end of the 20 th century. Thus the highest extreme-flood risk would increase $25 \%$ risks attributed from the 20 th century climate changes due to the increase of greenhouse gases; this could provide scientific basis for understanding and predicting the flood disasters responding to the global warming.
\end{abstract}

Keywords: Extreme flood effects; climate response; uncertainty; catchment hydrological modeling; attribution; Lake Taihu

随着全球气温显著增加, 人们观测到过去几十年的降水呈现明显的年代际变化 ${ }^{[1]}$, 而探究是否由人类 活动排放温室气体导致全球增温并造成极端降水和洪水变化,是一个极具挑战的理论问题. 近年来国内外 一些探索性研究认为, 北半球 50 年来最恶劣的暴雨和风雪事件呈上升趋势, 年均升幅约为 $7 \%$; 而通过气候 模拟研究,认为这些极端暴雨事件主要归因于温室气体作用下的全球增暖 ${ }^{[2]}$. 对 2000 年秋英国发生的特大

* 科技部全球变化重大科学研究计划项目 (2012CB956103-5)、中国科学院知识创新工程重要方向项目 (KZCX2-YW338 ) 和创新团队国际合作伙伴计划项目 (KZZD-EW-TZ-08) 联合资助. 2013-01-21 收稿; 2013-03-11 收修改 稿.于革,女,1957 年生, 研究员;E-mail: geyu@ niglas. ac. cn. 
洪水进行数值模拟, 发现温室气体排放增加造成了温度升高、极端降水增强, 引起特大地表洪水的发生由 100 年一遇加速到 50 年一遇 ${ }^{[3]}$. 这些研究揭示了增温引起降水和洪水的驱动机制并计算出所增加的份额, 然而由于时间尺度局限在近 50 年, 已经处于人类显著影响全球增温时段, 这类研究还难以辨识增温中的自 然系统下的脉动和变化份额. 寻找现代全球增温下与工业革命前大气状况下的极端洪水事件, 能够对比洪 水强度和频率变化的差异, 进而通过物理机制下的数值模拟能够获得对其差异的机制和归因认识 ${ }^{[1-3]}$.

特大洪水属于极端水文事件. 从统计上来讲, 极端洪水事件是指洪水发生的频率或重现期严重偏离其 平均态, 在统计意义上属于不易发生的事件. 根据现代水文观测数据, 重现期超过 50 年的洪水被称为特大 洪水 $^{[4]}$. 对超过洪水 50 年一遇的小概率事件, 常称为极端洪水 ${ }^{[1,3]}$. 长江下游太湖流域, 历史和近现代不乏 极端洪水发生 ${ }^{[5]}$. 新石器一陶器时期太湖古文化的延续曾遭受数次大洪水侵袭而发生过中断 ${ }^{[6]}$, 洪水之患自 古以来就是该区的最大威胁. 20 世纪发生了如 1954、1992 和 1999 年的特大洪水, 其中由气象和水文仪器监 测的 1999 年太湖流域洪水是自水文站监测以来的百年一遇的特大洪水 ${ }^{[7]}$. 根据历史文献记载, 19 世纪发生 了如 1823、1849 和 1889 年的特大洪水 ${ }^{[8.9]}$. 有研究表明, 采用相同水位标准估计 1889 年洪水水位比 1954 年 洪水水位高出 $6 \mathrm{~cm}^{[9]}$. 太湖流域 19 世纪末和 20 世纪末不同气候背景下的极端洪水事件, 为洪水归因研究 提供了难得的参照系.

我国东部长江中下游平原地势平坦, 属亚热带季风气候, 自古以来只要中、上游降雨过量, 下游平原就 出现水患 ${ }^{[4]}$. 现代太湖流域尽管人类围坓、建坝、土地利用等在一定程度上造成湖泊水体容量减少, 或下游 排水不畅导致洪水量的相对增大, 但降水的增减仍然是流域洪水最重要的水源量 ${ }^{[7,10-11]}$. 因此, 无论现代还 是历史, 降水都是控制流域洪水最重要的因素. 在这个立论下, 本文针对 $1990 \mathrm{~s}$ 和 $1880 \mathrm{~s}$ 的极端洪水历史, 设 计了太湖流域两个洪水年模式, 在 IPCC 诊断的温室气体变化的方案下, 采用 4 个 GCM 气候模拟驱动流域 水文模拟, 分析不同气候背景下极端洪水发生频率的差异, 并进行极端流量不确定性的阈值模拟和风险系 数论证, 从而检测不同气候驱动下的洪水归因.

\section{1 数据和方法}

\section{1 区域资料和模拟实验设计}

太湖平均水位面积 $2338 \mathrm{~km}^{2}$, 流域面积 $36895 \mathrm{~km}^{2}$. 考虑到太湖为通江湖泊以及旨在洪水的气候归因研 究, 本研究集中于太湖流域上游来水区, 主要位于西部山丘区和北部沿江区, 模拟面积 $19055 \mathrm{~km}^{2}$. 流域地 形、水系、植被、土壤等自然地理资料来自于多年积累的流域基础数据库 ${ }^{[12]}$. 流域气象数据源于国家气象台 站观测资料,包括流域内气象站 1954-2009 年逐日平均降水、最高与最低气温、相对湿度和风速. 太阳辐射 要素由最高、最低气温资料推算. 作为率定模型参数和验证控制实验的水文资料, 采用太湖大浦站 $1922-$ 2009 年实测水位资料 ${ }^{[11,13]}$ 、流域内河道巡测断面 1988- 2002 年流量 ${ }^{[12-13]}$ 和太湖特大洪水资料 ${ }^{[7,14]}$.

太湖是一个大型浅水湖泊, 流域大部分的平原地势平坦, 河床比降小, 流速缓, 流域输人太湖的输沙量 在 $30 \sim 40$ 万吨级; 而由于太湖流域雨量丰沛, 年降水量 $1100 \sim 1400 \mathrm{~mm}$, 人湖年径流量在 $40 \times 10^{8} \mathrm{~m}^{3}$ 以 上 $^{[15]}$, 因此, 太湖洪水变化主要受流域径流输人控制. 本文采用分布式流域水文模型 SWAT $2005^{[16]}$. 对以湖 泊水位和湖面高度变化为表征的产流、汇流的模拟研究已表明,SWAT 能够胜任该流域的水文模拟 ${ }^{[12]}$. 对长 期气候水文过程变化, 笔者对过去 200 年以来的模拟研究, 也表明 SWAT 能够模拟流域水量长期的年内和 年际变化 ${ }^{[17]}$. 因此,围绕洪水的气候归因研究目标, 笔者设计了采用该流域水文模型的模拟实验:

1) 控制实验: 太湖流域地形、水文、气候、植被、土壤等现代条件下的水文模拟实验,测试流域水文模型 对太湖洪水的模拟能力;2) 实验 $1: 1990 \mathrm{~s}$ 极端洪水模拟, 采用 GCM 气候模式驱动模拟 15 年 (1988-2002 年), 包括 1999 年特大洪水年的模拟;3) 实验 $2: 1980 \mathrm{~s}$ 极端洪水模拟, 采用 GCM 气候模式驱动模拟 15 年 $(1880-1894$ 年),包括 1889 年特大洪水年的模拟.

控制实验是本项研究的基础,该实验的模拟过程和结果见文献 [18]. 对该模拟结果的检验 ${ }^{[18]}$ 表明,在 气候驱动下, 该模型对现代或对历史的特大洪水具有较好的模拟能力, 能够捕捉到小概率水文事件. 因此, 本文主要介绍实验 1 和实验 2 的工作, 但采用控制实验的结果作为基线, 检查实验 1 和 2 由 GCM 驱动的水 文变化. 模型 SWAT 在 ArcView 界面上运行, 输出变量采用流域汇人太湖的逐日和逐月流量 $(Q)$, 并计算年 
平均流量、 $0.1 \%$ 和 $0.5 \%$ 的极端洪水流量 $\left(Q_{0.1 \%}\right.$ 和 $\left.Q_{0.5 \%}\right)$.

\section{2 极端洪水年的气候模式}

根据《政府间气候变化专业委员会》第四次评估报告( IPCC-AR4) 评估的过去 150 年来温室气体排放量 方案 $(\text { SRES })^{[1]}$, 国际上多个气候模式组织采用 SRES 方案进行了全球大气环流模式 (GCM) 的气候模拟实 验. 目前在 SRES 方案下模拟过去 150 年以来的气候变化,有 16 个 GCM 被 IPCC-AR4 数据中心收录并发 布 ${ }^{[19]}$. 根据应用经验 ${ }^{[20-21]}$, 本文选取了模式质量和精度相对较高、对东亚气候模拟相对适宜的海洋一生物一 冰圈与大气环流耦合的 GCM, 包括了英国 $\mathrm{HadCM}^{[22]}$ 、德国 $\mathrm{ECHAM}^{[23]}$ 、美国 $\mathrm{GFDL}^{[24]}$ 和加拿大 $\mathrm{CGCM}^{[25]} 4$ 个 模式. 这 4 个 GCM 的空间分辨率各异, HadCM3 为格点模式, 地表分辨率为 $2.75^{\circ}$ 纬度 $\times 3.75^{\circ}$ 经度; ECHAM5 为谱模式 T63 结构,地表分辨率为 $1.875^{\circ}$ 纬度 $\times 1.875^{\circ}$ 经度; GFDL2 为谱模式 R30 结构, 地表分辨 率为 $2.0^{\circ}$ 纬度 $\times 2.5^{\circ}$ 经度; CGCM3 为谱模式 T47 结构, 地表分辨率为 $3.75^{\circ}$ 纬度 $\times 3.75^{\circ}$ 经度. 在 $\mathrm{GCM}$ 全球 输出中采用包含太湖流域在内的区域、地理位置围绕 $110^{\circ} \sim 120^{\circ} \mathrm{E}$ 和 $28^{\circ} \sim 32^{\circ} \mathrm{N}$ 范围的纬向和经向 12 个网 格 $(3 \times 4)$.

针对 19 世纪和 20 世纪不同气候归因下的洪水发生, 本文采用 IPPC-AR4 两个方案下的气候模拟 ${ }^{[1,19]}$ 驱 动流域水文模拟:

1) 20C3M 方案:采用 1850 年以来观测到的大气温室气体增加状况进行气候模拟,代表了 19-20 世纪 真实的大气温室气体变化, 其中 $\mathrm{CO}_{2}$ 浓度从工业革命前 $280 \mathrm{mg} / \mathrm{L}$ 增加到 2005 年的 $379 \mathrm{mg} / \mathrm{L}, \mathrm{CH}_{4}$ 浓度从 $715 \mu \mathrm{g} / \mathrm{L}$ 增加到 $1774 \mu \mathrm{g} / \mathrm{L}$. 因此, 该气候模拟从机理上反演 20 世纪温室气体实际增加下的气候变化. 在此 方案下, HadCM 模拟了 1850 年以来 140 模式年,其他 3 个模式 ECHAM 、GFDL 和 CGCM 分别采用 1860 、1861 和 1850 年以来的 150 模式年. 考虑到该方案下的模拟系温室气体 150 年来随着时间的实际增长变化, 本文 采用了 $1988-2002$ 年 15 年气候模拟数据驱动实验 1 .

2) PICTL 方案: 采用工业革命前 1850 年大气温室气体作为常数对过去 150 年的气候模拟,代表了从机 理上设置的 20 世纪温室气体未增加、工业革命前自然大气温室气体下的气候状况, 其中 $\mathrm{CO}_{2}$ 和 $\mathrm{CH}_{4}$ 大气浓 度分别采用 $280 \mathrm{mg} / \mathrm{L}$ 和 $715 \mu \mathrm{g} / \mathrm{L}$. HadCM 、ECHAM 、GFDL 和 CGCM 在 PICTL 方案下分别模拟了自 1859 、 $1860 、 1860$ 和 1850 年开始的 $340 、 505 、 500$ 和 500 模式年. 本文采用了围绕 1889 年前后的 15 年气候模拟数 据驱动实验 2 .

\section{3 不确定性分析}

采用的 GCM 模拟的气候数据进行流域水文模拟, 主要存在以下 3 方面的不确定性:

1) 气候模式误差:包括不同 GCM 模拟实验对温室气体排放的大气层次、温室气体的光学物理和化学效 应等不同设置、模式对大气环流和大气浓度不同系统的响应以及模式自身的系统误差. 对此, 本文采用 4 个 GCM 的多模式集合法 ${ }^{[26]}$, 以减少采用单个模式的误差.

2) 全球空间尺度的 GCM 应用到太湖流域的区域误差: 尽管根据应用经验选取了 4 个比较优秀的 $\mathrm{GCM}$, 但这些欧美气候模式对东亚气候系统把握程度各异, 应用到太湖流域可产生一定的不确定性 ${ }^{[27-28]}$. 因 此, 本文采用与观测资料序列拟合与校验的统计降尺度方法 ${ }^{[29]}$, 校正区域误差.

在逐日降水的随机模拟中, 采用一阶马尔科夫链和和分布函数相结合证明可较好地模拟逐日降水过 程 ${ }^{[30-34]}$. 本文应用该方法, 首先用马尔科夫链描述降水日的发生, 再用选定的分布函数拟合降水日的降水 量,并采用实测数据予以验证. 基本步骤如下:

i) 首先根据 1950-2000 年太湖降水的实测数据, 建立一阶马尔科夫链模型的两个基本状态 (即降水日 $\mathrm{W}$ 和非降水日 $\mathrm{D})$ 数据矩阵. 然后, 计算从 2 周内至年内的转移概率, 即降水日到降水日转移概率 $\mathrm{P}(\mathrm{WW})$ 和 非降水日到降水日转移概率 $\mathrm{P}(\mathrm{WD})$, 使所分析的序列具有马尔科夫链的性质.

ii) 选定的分布函数拟合降水日的降水量. 由于本文研究极端值的变化,没有采用常用的 Gamma 分布函 数 ${ }^{[32-34]}$, 而是采用了能较好描述极端值分布型的对数正态分布函数 ${ }^{[30-31]}$. 这个分布型经过了论证和检验适 合太湖极端年份的降水分布 ${ }^{[18]}$. 由此估计出对数正态分布的位置参数 $(\mu)$ 和尺度参数 $(\sigma)$, 从而实现逐日 降水的模拟.

iii) 利用实测降水数据对极端年份 (1999 年) 的逐日降水模拟进行了统计检验. 结果表明, 实测值与 4 
个 GCM 模拟值的相关系数分别为 0.242 (HadCM)、0.229 (ECHAM)、0.202 (GFDL) 和 0.327 (CGCM). 在该 极端年份的样本下 $(n=365)$, 总体相关系数检验 $P<0.05$, 表明两个变量呈线性相关.

iv）该方法分别应用 4 个 GCM 在 PICTL 和 20C3M 方案下的逐月降水模拟,计算出太湖流域逐日降水 序列.

3) 有限样本认识极端值的误差: 根据极端值理论, 在认识极端水文事件的总体分布不完全的情况下, 分 析流量极端值存在着很大的不确定性. 采用以下步骤进行随机统计模拟和不确定性阈值限定:

i) 由于流量分布呈近似不对称型, 可采用与降水相似、接近对数正态分布 ${ }^{[31]}$ 的拟合. 可利用流量序列 分布的位置参数 $(\mu)$ 和尺度参数 $(\sigma)$ 验证是否服从对数正态分布 $\sim \log -\operatorname{Normal}(\mu, \sigma)$, 并采用 KolmogorovSmirnov 单样品序列检验 $(\text { KS-test1 })^{[35]}$;

ii) 采用蒙特卡罗方法 ${ }^{[3,28]}$ 对有限的 15 年样本序列按照正态对数分布进行大样本模拟;

iii）采用 Bootstrap 方法 ${ }^{[36]}$ 对有限的 15 年样本序列进行置信区间模拟, 从而限定其不确定性的统计 范畴;

iv) 根据论证的洪水偏态分布, 采用 Kolmogorov-Smirnov 双样品序列检验 (KS-test2) ${ }^{[35]}$ 对不同模式的结 果进行差异性统计检验.

\section{4 极端洪水的气候效应和风险系数}

为了诊断温室气体变化下气候模式对洪水的影响, 构建一个风险系数 $F$ 值 ${ }^{[37-38]}$ 用来定量描述太湖流域 洪水在 20 世纪末与 19 世纪末的气候效应, 并估测工业革命前气候与 20 世纪末气候所产生洪水风险的差 异. $F$ 值采用 PICTL 方案下模拟的流量 $\left(Q_{\text {PICTL }}\right)$ 与 $20 \mathrm{C} 3 \mathrm{M}$ 方案下模拟的流量 $\left(Q_{20 \mathrm{CBM}}\right)$ 之比:

$$
F=1-Q_{\text {PICTL }} / Q_{20 \text { сзм }}
$$

$F$ 值无量纲, 当 $F$ 值 $<0$ 时, 表示 20 世纪末的洪水风险减少, 从 0 减少到最小值; 当 $F$ 值 $>0$ 时, 表示 20 世纪末的洪水风险增加, 从 0 增加到最大值. 为了避免 $F$ 值中分母为 0 , 在 $Q_{\mathrm{PICTL}}$ 和 $Q_{20 \text { с } 3 \mathrm{M}} 15$ 年的时间序列 $(n=5830 \mathrm{~d})$ 中, 对流量标准化:

$$
Q_{\mathrm{s}}=2\left(Q-Q_{\min }\right) /\left(Q_{\max }-Q_{\min }\right)-1
$$

式中, $Q_{\mathrm{s}}$ 和 $Q$ 分别是标准化后和标准化前的流量, $Q_{\text {min }}$ 和 $Q_{\text {max }}$ 分别是标准化前最小和最大流量. 这样, 计算 $F$ 值在 $(-\infty, 1)$ 之间. 尽管这个数据序列是一个不对称分布, 可以通过计算风险系数在总体分布中 $(-\infty, 1)$ 的百分位 $(\%)^{[3]}$, 以查看洪水风险的相对增减变化.

上述 1.3 和 1.4 节的数据矩阵和模式程序均在 Matlab 界面上处理、运行和计算.

\section{2 模拟结果}

\section{1 现代气候模式的洪水模拟 ( 实验 1 )}

在实验 1 中, 采用 IPCG-20C3M 方案的大气温室气体水平气候背景下 4 个 GCM 对 1988-2002 年气候 模拟驱动, 模拟太湖流域汇流过程, 获得流域汇人太湖的逐日流量. 在模拟的 15 年中, HadCM、 ECHAM、 GFDL、CGCM 的平均流量分别为 $182.1 、 181.6 、 167.8 、 171.9 \mathrm{~m}^{3} / \mathrm{s}$. 与 $1990 \mathrm{~s}$ 观测到的平均流量 $\left(183 \mathrm{~m}^{3} / \mathrm{s}\right)$ 和 控制实验平均流量 $\left(185 \mathrm{~m}^{3} / \mathrm{s}\right)$ 相比, 这些模拟比较接近观测值. 由 HadCM、ECHAM、GFDL、CGCM 4 个 GCM 驱动,均模拟出 1999 年的极端洪水,其降水过程和模拟的洪水过程见图 1.

对 1988-2002 年气候驱动的水文模拟计算 $0.1 \%$ 的洪水流量, 4 个 GCM 模拟范围为 $2930 \sim 3603 \mathrm{~m}^{3} / \mathrm{s}$, $0.5 \%$ 的洪水流量模拟范围为 $1843 \sim 1894 \mathrm{~m}^{3} / \mathrm{s}$ ( 图 2a). 根据日最大流量瞬时实测的记录, 1983 年 7 月 3 日 是 $2770 \mathrm{~m}^{3} / \mathrm{s}, 1991$ 年 6 月 5 日是 $2450 \mathrm{~m}^{3} / \mathrm{s}, 1999$ 年 6 月 28 日是 $3029 \mathrm{~m}^{3} / \mathrm{s}^{[7]}$. 实验 1 模拟的多年最大值达 到其上限, 认为可信. 与控制实验模拟的小于 $5 \%$ 洪水流量 $\left(Q_{\text {extreme }}\right)$ 相比, HadCM、ECHAM、GFDL、CGCM 的 均方根误差分别为 $182 、 191 、 186 、 262 \mathrm{~m}^{3} / \mathrm{s}$, 相对误差分别为 $7.8 \% 、 10.3 \% 、 8.5 \% 、 8.1 \%$, 均在 $10.3 \%$ 以下, 属经验可接受范围. 此外, Bootstrap 置信区间模拟, 在 $P<0.05$ 水平上 $1990 \mathrm{~s}$ 流量均在控制实验的误差区域 之内 (图 2a). 


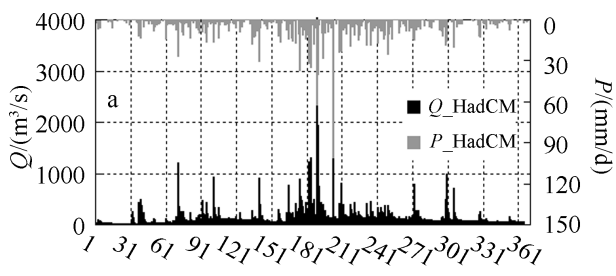

时间 $/ \mathrm{d}$

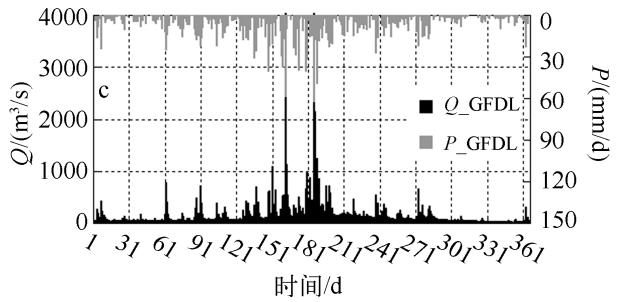

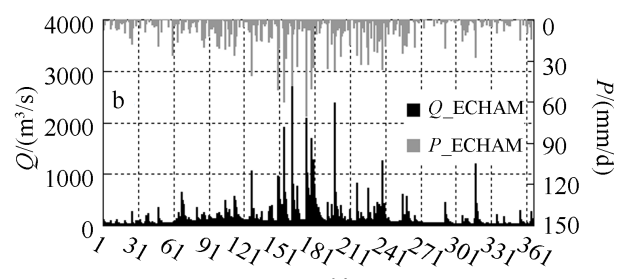

时间/d

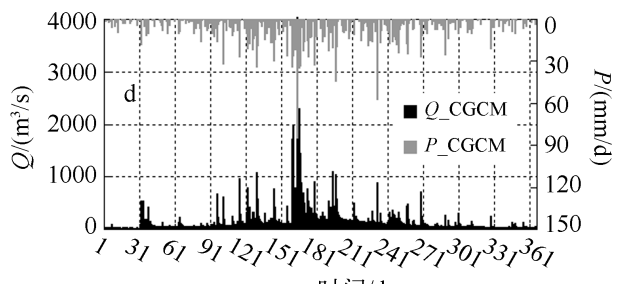

时间 $/ \mathrm{d}$

图 14 个 GCM 在 IPCC-20C3M 方案下 1999 年逐日降水 $(P)$ 过程和 所驱动的流域水文实验 1 的逐日流量 $(Q)$ 过程

Fig. 1 Daily precipitation $(P)$ simulations driving by four GCM simulations of IPCC-20C3M scenarios, and the driving 1999 daily discharge $(Q)$ simulations in Exp. 1

\section{2 历史气候模式的洪水模拟 (实验 2 )}

在实验 2 中,采用 IPCC-PICTL 方案的工业革命前大气温室气体水平气候背景下 4 个 GCM 对 $1880-$ 1894 年气候模拟, 模拟太湖流域汇流过程, 获得流域汇人太湖逐日流量 (图 3a、b、c、d). 在模拟的 15 年中, HadCM 、ECHAM GFDL、CGCM 的平均值分别为 $175.8 、 165.7 、 149.1 、 171.8 \mathrm{~m}^{3} / \mathrm{s}$. 模拟的流量逐日变化分布 与各自 GCM 模拟的降水分布基本一致(图 3e、f、g、h).

为了分析洪水极端值变化, 画出在 4 个 GCM 驱动的 15 年流域水文序列模拟中 $\leqslant 5 \%$ 的逐日洪水流量频 率分布图 (图 2b). HadCM、ECHAM、GFDL、CGCM 驱动模拟的 $0.1 \%$ 的洪水流量范围为 $2069 \sim 3119 \mathrm{~m}^{3} / \mathrm{s}$, $0.5 \%$ 的洪水流量范围为 $1437 \sim 1561 \mathrm{~m}^{3} / \mathrm{s}$. 与控制实验相比, 可以观查到 4 个 GCM 气候驱动的极端流量 $\left(Q_{\text {extreme }}\right)$ 为 $0.01 \% \sim 2.00 \%$ 部分显著降低. Bootstrap 置信区间模拟的 $P<0.05$ 范围显示, 1880 s 流量已经在 控制实验的误差区域之外,可直观看到与控制实验的显著差异.

\section{3 分析和讨论}

为了分析不同气候模式对极端洪水的影响, 对 IPCC-PICTL 和 IPCC-20C3M 两个方案下 GCM 气候模拟 驱动的流域水文模拟进行对比. 对实验 1(1988-2002 年) 和实验 2(1880-1894 年) 模拟的逐日流域流量输 出,KS-test2 检验表明,4 个 GCM 的每两对方案下流量序列均具有显著差异 $(P<0.017)$. 主要统计量 (表 1) 表明,工业革命前大气温室气体状况下的平均流量 $\left(165 \sim 196 \mathrm{~m}^{3} / \mathrm{s}\right)$ 比 1990s $\left(192 \sim 215 \mathrm{~m}^{3} / \mathrm{s}\right)$ 偏小, 标准偏 差也偏小. 从极端值来看, $Q_{0.1 \%}$ 和 $Q_{0.5 \%}$ 的流量 $\left(2069 \sim 3119 \mathrm{~m}^{3} / \mathrm{s}\right.$ 和 $1436 \sim 1561 \mathrm{~m}^{3} / \mathrm{s}$ ) 均比 $1990 \mathrm{~s}(2929 \sim$ $3601 \mathrm{~m}^{3} / \mathrm{s}$ 和 $1842 \sim 1893 \mathrm{~m}^{3} / \mathrm{s}$ ) 显著偏小.

为了对比工业革命前 (PICTL 方案) 与 20 世纪末 (20C3M 方案) 4 个 GCM 气候驱动下的洪水响应的差 异,笔者采用样本 $n=10000$ 服从正态对数分布的蒙特卡罗随机抽样模拟, 以检查洪水流量在大样本下的极 端值 (图 4a、b、c、d) 和分布特征(图 4e、f、g、h). 流量模拟的 4 个 GCM 的 PICTL 模式在极端值区间比 20C3M 模式显著偏小 (图 4), 反映出大气温室气体在工业革命前的气候状况下, 太湖流域极端流量比 20 世纪末显 著减少 (KS-test2 检验, $P<0.01$ ).

检查 IPCC-PICTL 方案和 20 C 3 M 方案下 4 个 GCM 气候模拟驱动的太湖洪水的发生风险, 采用了风险系 数 ( $F$ 值) 定量描述上述 “偏小”特征. 根据公式 $(1)$ 和 $(2)$ 计算 4 个 GCM 在 PICTL 方案和 $20 \mathrm{C} 3 \mathrm{M}$ 方案驱动洪 水模拟的 $F$ 值, 做出 $F$ 值频率分布图 (图 5), 可以看到 HadCM 、ECHAM、GFDL、CGCM 这 4 个模式的最大频 率分别为 $23 \%(F=0.6) 、 14 \%(F=0.6) 、 12 \%(F=0.2)$ 和 $18 \%(F=0.6), 4$ 个 GCM 洪水风险系数出现频 
a
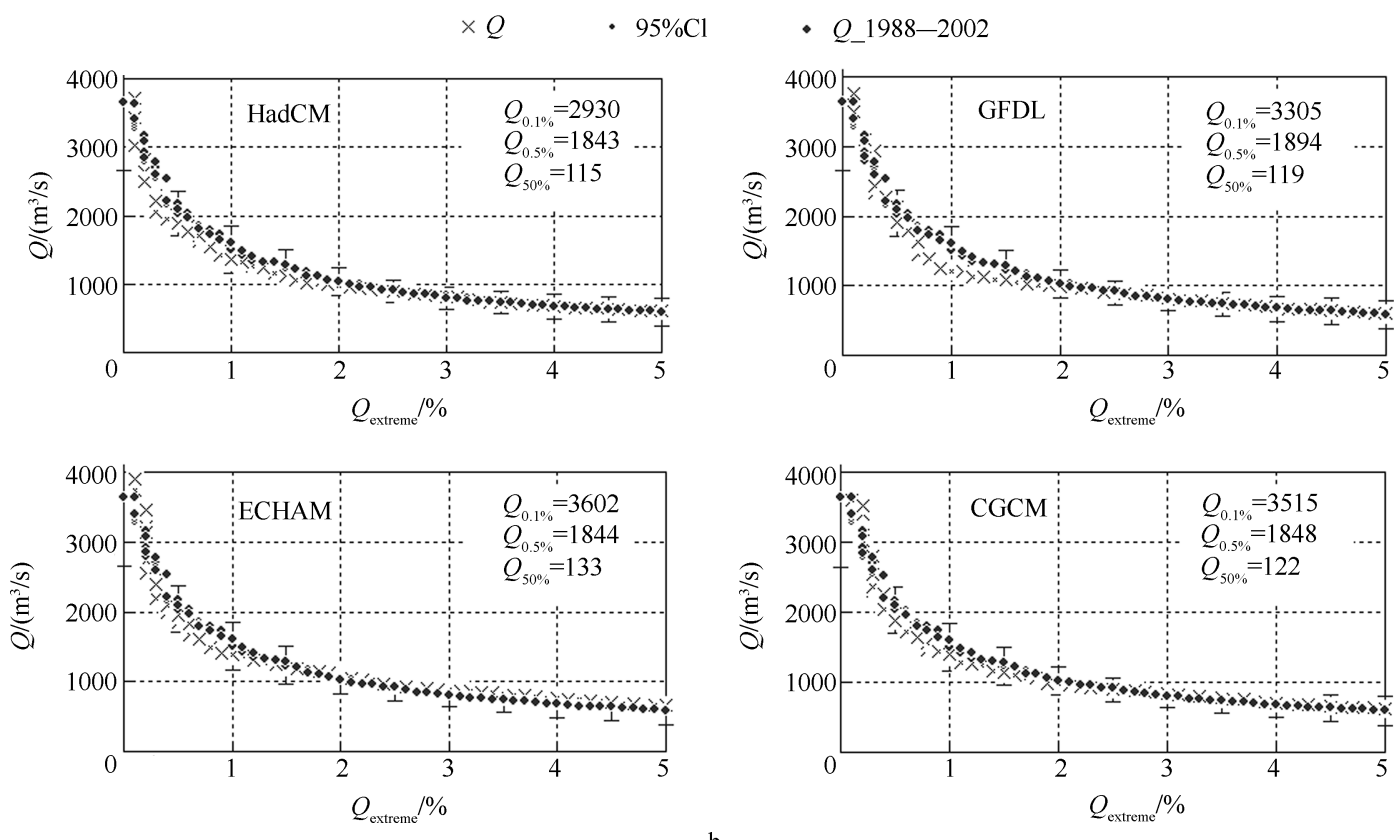

b

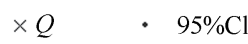

- Q_1988-2002
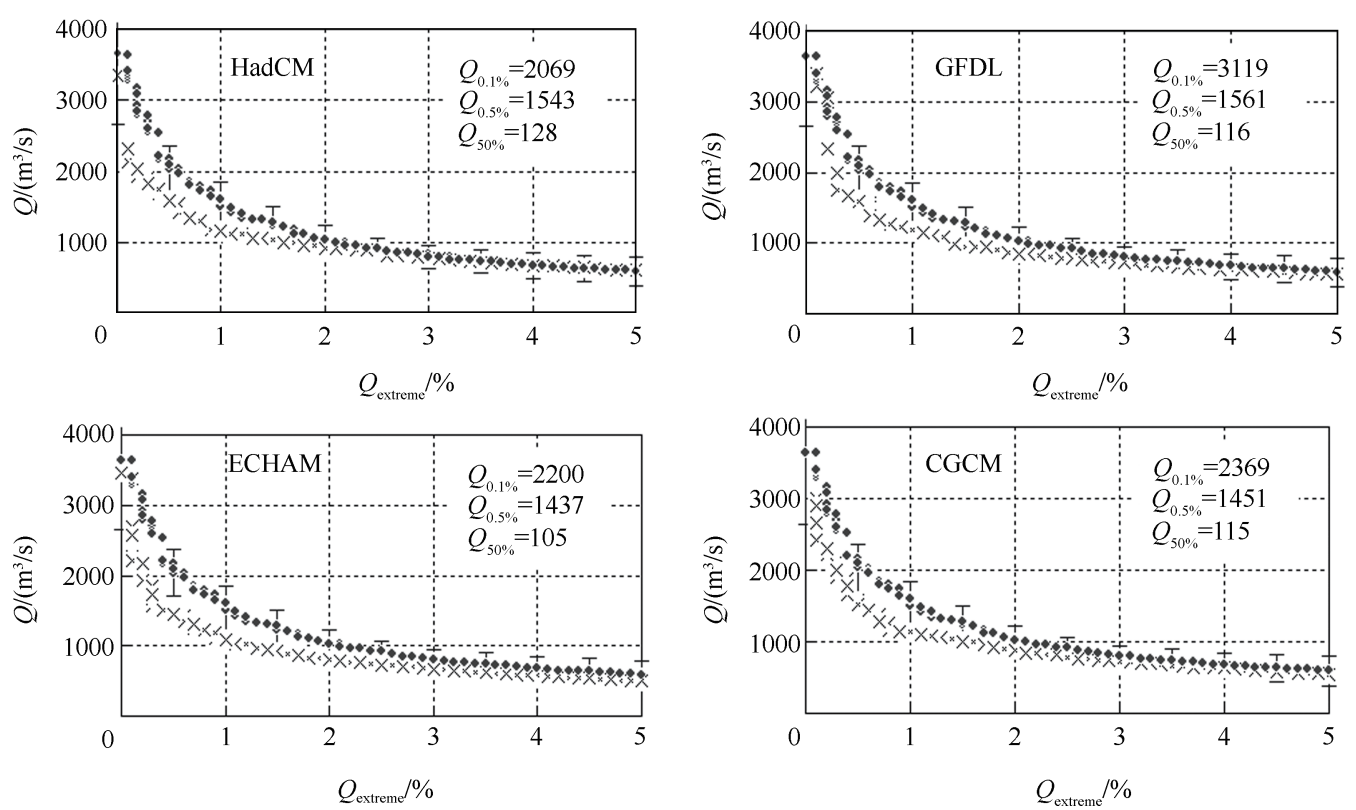

图 24 个 GCM 在 IPCC-20C3M 方案 (a) 和 IPCC-PICTL 方案 (b) 下驱动的流域水文 模拟的逐日流量极端值频率 $\left(Q_{\text {extreme }}<5 \%\right)$ 分布, 并与控制实验的流量 $\left(Q \_1988-2002\right)$ 对比 (蓝色误差范围为 Bootstrap 模拟的置信区间 $(P<0.05))$

Fig. 2 Frequencies of daily discharge extremes $\left(Q_{\text {extreme }}<5 \%\right)$ by the hydrological simulations driving by four GCM simulations of IPCC-20C3M(a) and IPCC-PICTL(b) scenarios, and comparing with discharge simulations ( $\left.Q \_1988-2002\right)$ in the control run(Blue error bars are the 

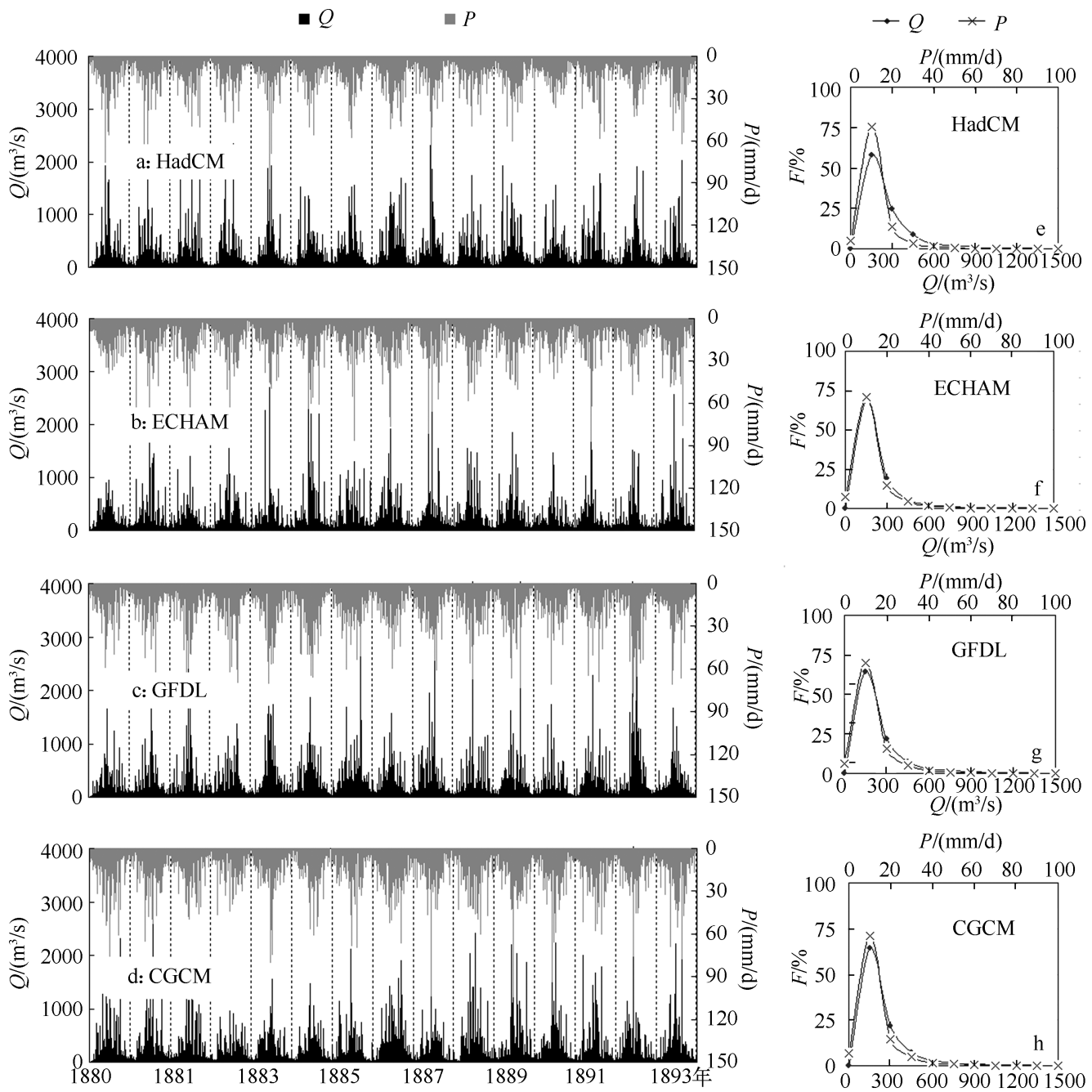

$P /(\mathrm{mm} / \mathrm{d})$

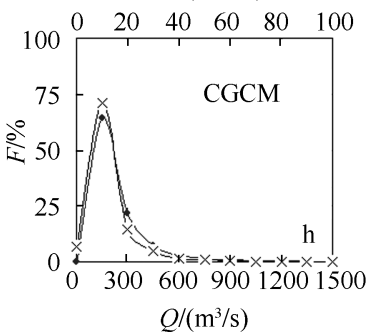

图 34 个 GCM 在 IPCC-PICTL 方案下逐日降水 $(P)$ 过程模拟和流域水文实验 2 的 15 年逐日流量 $(Q)$ 过程模拟 $(a, b 、 c 、 d)$, 以及相应降水和流量的频率分布 $(e 、 f 、 g 、 h)$

Fig. 3 Daily precipitation $(P)$ simulations driving by our GCM simulations of IPCC-PICTL scenarios and the discharge $(Q)$ simulations in Exp. 2 ( $\mathrm{a}, \mathrm{b}, \mathrm{c}$ and $\mathrm{d})$. Frequencies of the precipitation $(P)$ and discharge $(Q)$ were plotted respectively in Figs. e, $\mathrm{f}, \mathrm{g}$ and $\mathrm{h}$

率最大值都为正值, 代表着洪水风险的增加. 计算各个序列的中值 (50\% 百分位) 可以查看该组数据中 小于和大于一半的数据位居何处, 结果显示 HadCM、ECHAM、GFDL、GGCM4 个 GCM 气候驱动下的风 险系数的中值分别为 $0.44 、 0.15 、-0.09 、 0.28$, 表明除了 GFDL 模拟的流量风险系数中值接近于 0 外,其他 3 个模式风险系数的中值均处于正值 $0.15 \sim 0.44$, 反映了洪水风险的显著增加. 由于计算 $F$ 值界于 $[-50,1]$ 中, 呈不对称分布, 可以通过计算风险系数在总体分布中 $[-50,1]$ 的百分位 ${ }^{[3]}$, 查 看风险的相对增减变化. 结果显示, HadCM 、ECHAM、GFDL、CGCM 4 个模式驱动的频率最大的洪水风 险分别是 $60 \% 、 40 \% 、 20 \%$ 和 $40 \%$ （图 5 ), 反映出 20 世纪末的洪水流量变化较 19 世纪的风险显著增加 $20 \% \sim 60 \%$. 
表 1 实验 1(1988-2002 年) 和实验 2(1880-1894 年) 流域人湖流量模拟的主要统计量

Tab. 1 Major statistics of discharge simulations from Exp. 1 and Exp. 2

\begin{tabular}{ccccccc}
\hline 气候模式 & 实验 & 模拟方案 & 平均值 $/\left(\mathrm{m}^{3} / \mathrm{s}\right)$ & 标准偏差 $/\left(\mathrm{m}^{3} / \mathrm{s}\right)$ & $Q_{0.1 \%} /\left(\mathrm{m}^{3} / \mathrm{s}\right)$ & $Q_{0.5 \%} /\left(\mathrm{m}^{3} / \mathrm{s}\right)$ \\
\hline HadCM & 实验 1 & $20 \mathrm{C} 3 \mathrm{M}$ & 192.8 & 271.5 & 2929.6 & 1842.8 \\
HadCM & 实验 2 & PICTL & 196.4 & 229.3 & 2069.1 & 1543.2 \\
ECHAM & 实验 1 & $20 \mathrm{C} 3 \mathrm{M}$ & 198.4 & 279.3 & 3305.0 & 1893.4 \\
ECHAM & 实验 2 & PICTL & 184.8 & 243.4 & 3119.3 & 1561.2 \\
GFDL & 实验 1 & 20C3M & 203.4 & 291.0 & 3515.4 & 1847.8 \\
GFDL & 实验 2 & PICTL & 179.8 & 232.7 & 2369.3 & 1451.3 \\
CGCM & 实验 1 & 20C3M & 215.2 & 288.9 & 3601.9 & 1843.6 \\
CGCM & 实验 2 & PICTL & 165.7 & 210.0 & 2200.4 & 1436.4
\end{tabular}
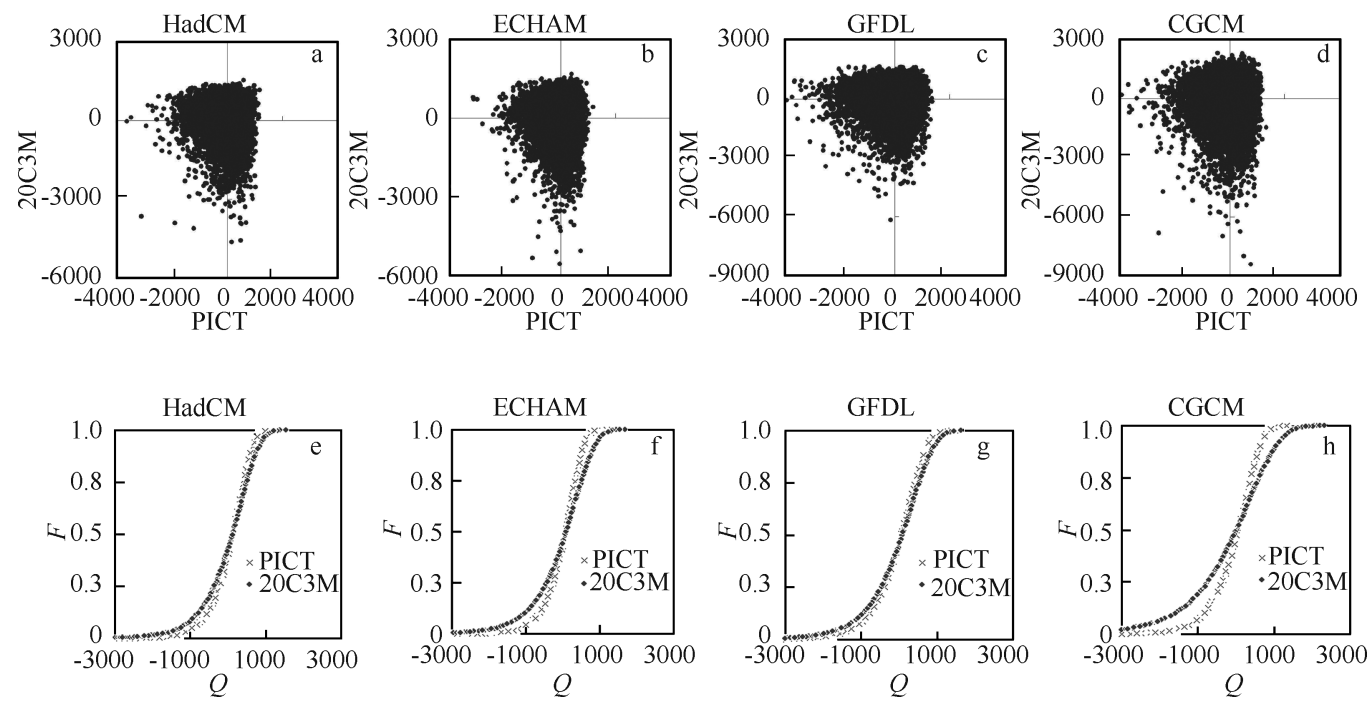

图 4 蒙特卡罗模拟太湖流域不同时间逐日流量变化的频数变率分布 $(\mathrm{a} 、 \mathrm{~b} 、 \mathrm{c} 、 \mathrm{~d}$ : 横坐标 PICT 代表 19 世纪末,

纵坐标 20C3M 代表 20 世纪末) 以及洪水风险系数 $(F)$ 与频数变率 $(Q)$ 的分布 $(\mathrm{e} 、 \mathrm{f} 、 \mathrm{~g} 、 \mathrm{~h})$, 分别对比 4 个 GCM 工业革命前 (PICTL 方案) 与 20 世纪末 (20C3M 方案) 气候驱动下的洪水效应

Fig. 4 Frequency variability of daily discharge ( a, b, c and d: the end of the 19th century in the horizontal axis and the end of the 20th century in the vertical axis) and flood risk ratio $(F)$ vs. the frequency variability $(Q)$ ( e, f, g and h) by Monte Carlo simulations in the Lake Taihu catchment, comparing with flood effects of climate changes driving by four GCM simulations of the pre-industry time (PICTL scenario) and the 20th century (20C3M scenario), respectively

\section{4 讨论和结语}

关于对全球增温是否能够引起降水增加超过气候统计意义上的变率、从而影响到极端洪水发生的认 识, 由于全球增温与气候系统脉动和振荡引起的温度变化互为交织, 判识导致温室气体是否导致自然灾害 加剧作用极为复杂, 是灾害发生预测和对策研究中急需解决的瓶颈. 此外, 由于我国大部分观测资料时间开 始于 1950s, 研究百年一遇以上的极端洪水受到时间长度的限制, 存在着很大的不确定性. 本文采用物理机 制下的数值模拟与随机系统模拟结合对此进行了探讨, 前者从气候驱动机制上模拟不同大气温室气候状况 下的洪水结果, 而后者旨在减少 GCM 驱动流域水文模拟过程中的不确定性. 采用 1980s 包括 1889 年特征洪 水年模式 (农耕时代的流域系统下、自然的大气环流驱动模式) 与 1990s 包括 1999 年特征洪水年模式 (20 世 纪末流域系统下、全球增温下大气环流驱动模式) 进行对比, 能够较好地从机制认识洪水差异的归因, 并能 
够进行极端流量的随机统计模拟和风险系数 论证.

从模拟的洪水极端值来看, $1990 \mathrm{~s}$ 流域极端洪 水流量 $\left(Q_{0.1 \%}\right.$ 为 $2929 \sim 3601 \mathrm{~m}^{3} / \mathrm{s}, Q_{0.5 \%}$ 为 $1842 \sim$ $1893 \mathrm{~m}^{3} / \mathrm{s}$ ) 比工业革命前大气温室气体状况下的 洪水流量 $\left(Q_{0.1 \%}\right.$ 为 $2069 \sim 3119 \mathrm{~m}^{3} / \mathrm{s}, Q_{0.5 \%}$ 为 $1436 \sim 1561 \mathrm{~m}^{3} / \mathrm{s}$ ) 显著偏大 (KS-test2 检验, $P<$ $0.017), 4$ 个 GCM 驱动的洪水产生的最大的风险 在 $20 \% \sim 60 \%$ 范围内, 反映出 20 世纪末的洪水流 量变化较 19 世纪的风险显著增加.

本研究中针对气候变化采用的驱动流域水文 模拟,流域下垫面采用 20 世纪末的设置. 因此, $20 \%$ 60\% 的洪水增加风险估计包含了气候变化 (降水、温度等) 和流域下垫面变化 (水利工程、土 地利用等) 两方面因素. 尽管根据太湖流域研 究 ${ }^{[7,10-11]}$ 认为降水的增减是流域洪水最重要的控 制因素,但下垫面变化的贡献仍然不可忽略. 根据

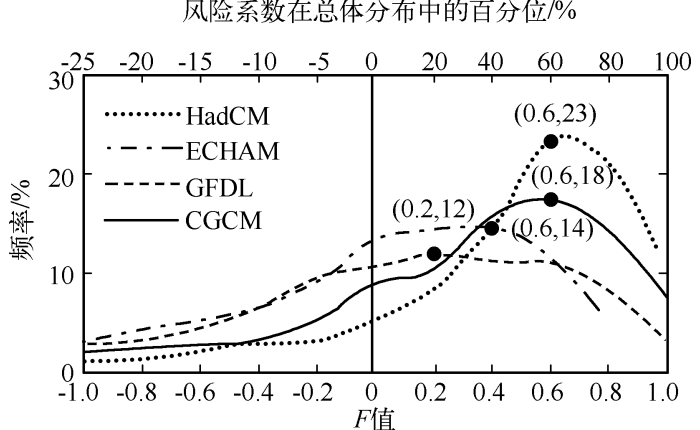

图 5 PICTL 方案和 $20 \mathrm{C} 3 \mathrm{M}$ 方案下 4 个 GCM 气候模拟驱动的太湖流量变化的风险系数 ( $F$ 值) 分布和风险增减在总体分布中的百分位 $(\%)$ ( 括号内数据表示最高频率的风险值和所占的频率)

Fig. 5 Frequencies of risk ratio ( $F$-ratio) and the increasing percentiles (\%) of the Lake Taihu extreme floods driving by four GCM simulations of PICTL and 20C3M scenarios 对 1999 年洪水灾害权重的研究,太湖围旺面积和 泥沙淤积使湖泊容积减少 $5.2 \%$,下游的淤塞使湖泊积水增加占 $24.3 \%$ 30. $2 \%^{[39]}$. 这两项均可以造成湖泊 洪水水位抬升和相对湖泊洪水量 (蓄洪量) 增加. 本文的太湖洪水效应来自于对入湖流量模拟,如果考虑湖 泊洪水的蓄洪量中有 30\% 35\% 变化,则由于 20 世纪末气候降水变化的 $20 \% \sim 60 \%$ 洪水增加应该减去该 项贡献,所以气候变化的洪水效应最大增量为 $25 \%$. 太湖流域 $1900 \mathrm{~s}$ 相对 $1880 \mathrm{~s}$ 下垫面变化从水文物理效应 上的贡献,有待今后进一步研究,包括通过敏感性因子的模拟实验认识在总体增加风险中、人工改造下垫面 因素的贡献份额.

大陆西岸的英国 2000 年秋季发生了百年一遇的极端洪水,经模拟研究认为由温室气体增加的气候变 化引起的洪水风险增加了 $50 \%{ }^{[3]}$. 太湖流域人类活动改变的下垫面在 1999 年特大洪水中引起最大增量约 $35 \%{ }^{[35]}$, 本文模拟和分析的 20 世纪末气候下的洪水最大增量占 $60 \%$,去除下垫面人为活动影响来估计洪 水增加的最大增量在 $25 \%$. 因此,认为在我国东部长江下游、太湖平原地区, 大气温室气体增加的气候变化 可引发极端洪水发生的风险增加. 这个认识有待进一步研究流域下垫面变化的水文物理效应, 从而为认识 与全球增温相关联的洪水灾害预测预警提供可靠的科学依据.

\section{5 参考文献}

[ 1 ] IPCC. The fourth assessment report of the intergovernmental panel on climate change. Cambridge: Cambridge University Press, 2007.

[ 2 ] Min SK, Zhang X, Zwiers FW et al. Human contribution to more-intense precipitation extremes. Nature, 2011, 470 : $378-381$.

[ 3 ] Pall P, Aina T, Dáithí A et al. Anthropogenic greenhouse gas contribution to flood risk in England and Wales in autumn. Nature, 2011, 470: 382-385.

[4] 中华人民共和国水利部. 水利水电工程等级划分及洪水标准. SL252-2000,2000.

[5] 胡明思,骆承政. 中国历史大洪水(下卷). 北京:中国书店出版社, 1988.

[6] 杨怀仁,谢志仁,杨达源. 全新世海面变化与太湖形成和演变. 见:杨怀仁编. 第四纪冰川与第四纪地质论文集. 北 京: 地质出版社, $1985: 49-64$.

[7] 欧炎伦, 吴浩云. 1999 年太湖流域洪水. 北京: 中国水利水电出版社,2001.

[8] 陈家其. 太湖流域历史洪水排队. 人民长江, 1992,32(2):30-33.

[9]《太湖水利史稿》编写组. 太湖水利史稿. 南京: 河海大学出版社,1993:118-122. 
[10］杨桂山,王德建. 太湖流域——经济发展・水环境・水灾害. 北京:科学出版社,2003.

[11] 罗潋葱,秦伯强,朱广伟. 太湖上游的水文特征及灾害分析. 灾害学,2004,19(1):21-26.

[12] 赖格英, 于 革, 桂 峰. 太湖流域营养物质输移模拟评估的初步研究. 中国科学: D 辑: 地球科学, 2005,35 (增刊 2) :121-130.

[13］中央水利部. 长江流域水文资料・太湖区. 南京: 中央水利部南京水利实验处,1951.

[14] 毛 锐.建国以来太湖流域三次大洪水的比较及对今后治理洪涝的意见. 湖泊科学,2000,12(1):12-18.

[15] 黄渏平,范成新,誉培民等.太湖水环境及其污染控制. 北京:科学出版社,2001.

[16] Di Luzio, Srinivasa MR, Arnold JG. A GIS-coupled hydrological model system for the Watershed assessment of agricultural nonpoint and point sources of pollution. Transactions in GIS , 2004, 8: 113-136.

[17] 于 革, 桂 峰. 太湖流域营养盐沉积 200 年的历史模拟研究. 沉积学报,2006,24(6):849-856.

[18］于 革,桂 峰,李永飞. 太湖 1899 洪水年的流域水文模拟. 湖泊科学,2012,24(6):651-657.

[19] IPCC Data Distribution Center. AR4 GCM data (Crown copyright 2005, Data provided by the Met Office Hadley Centre). http://www. ipcc-data. org, 2008.

[20] Yu G, Shen H, Liu J. Impacts of climate change on historical locust outbreaks in China. Journal of Geophysical Research-Atmospheres, 2009, 114: D18104 1-11.

[21] Yu G, Shen H. Lake water changes in response to climate change in northern China: Simulations and uncertainty analysis. Quaternary International, 2010, 212 : 44-56.

[22] Johns TC, Gregory JM, Ingram WJ et al. Anthropogenic climate change for 1860 to 2100 simulated with the HadCM3 model under updated emissions scenarios. Climate Dynamics, 2003, 20 : 583-612.

[23] Roeckner E, Brokopf R, Esch M et al. Sensitivity of simulated climate to horizontal and vertical resolution in the ECHAM5 atmosphere model. Journal of Climate, 2006, 19: 3771-3791.

[24] Manabe S, Stouffer RJ. Century-scale effects of increased atmospheric $\mathrm{CO}_{2}$ on the ocean-atmosphere system. Nature, $1993, \mathbf{3 6 4}(6434):$ :215-218.

[25] Flato GM, Boer GJ, Lee WG et al. The Canadian centre for climate modeling and analysis global coupled model and its Climate. Climate Dynamics, 2000, 16: 451-467.

[26] Semenov MA, Stratonovitch P. Use of multi-model ensembles from global climate models for assessment of climate change impacts. Climate Research, 2010, 41: 1-14.

[27] Moss R, Schneider S. Uncertainties in the IPCC TAR: Recommendations to lead authors for more consistent assessment and reporting. In: IPCC Supporting Material: Guidance Papers on Cross Cutting Issues in the Third Assessment Report of the IPCC. Geneva, 2000: 33-51.

[28] New M, Hulme M. Representing uncertainty in climate Change Scenarios: a Monte-Carlo approach. Integrate Assessment, $2000,(1)$ : 203-213.

[29] Hewitson BC, Crane RG. Climate downscaling: techniques and application. Climate Research, 1996, 7: 85-95.

[30 ] Hahn GJ, Shapiro SS. Statistical models in engineering. New York: John Wiley \& Sons, Inc. , 1967.

[31] Dingman SL. Physical hydrology : 2nd edition. New Jersey: Prentice Hall, 2002.

[32] 廖要明,张 强,陈德亮. 中国天气发生器的降水模拟. 地理学报,2004,59(5):689-698.

[33] 王 斌,付 强,王 敏等. 几种模拟逐日降水的分布函数比较分析. 数学的实践与认识,2011,41(9):128-133.

[34] 杨文峰,陈德亮,胡春娟. 天气发生器对陕西降水的模拟. 陕西气象,2005, (5):5-7.

[35 ] Marsaglia G, Tsang WW, Wang J. Evaluating Kolmogorov's distribution. Journal of Statistical Software, 2003,8 : 14.

[36] Efron B, Tibshirani RJ. An Introduction to the Bootstrap. Florida: Chapman \& Hall, 1993.

[37] Allen MR. Liability for climate change. Nature, 2003, 421: 891-892.

[38 ] Stone DA, Allen MR. The end-to-end attribution problem: from emissions to impacts. Climate Change, 2005 , 71 : 303 318.

[39] 秦伯强,胡维平,陈伟民等. 太湖水环境演化过程与机理. 北京:科学出版社,2004. 